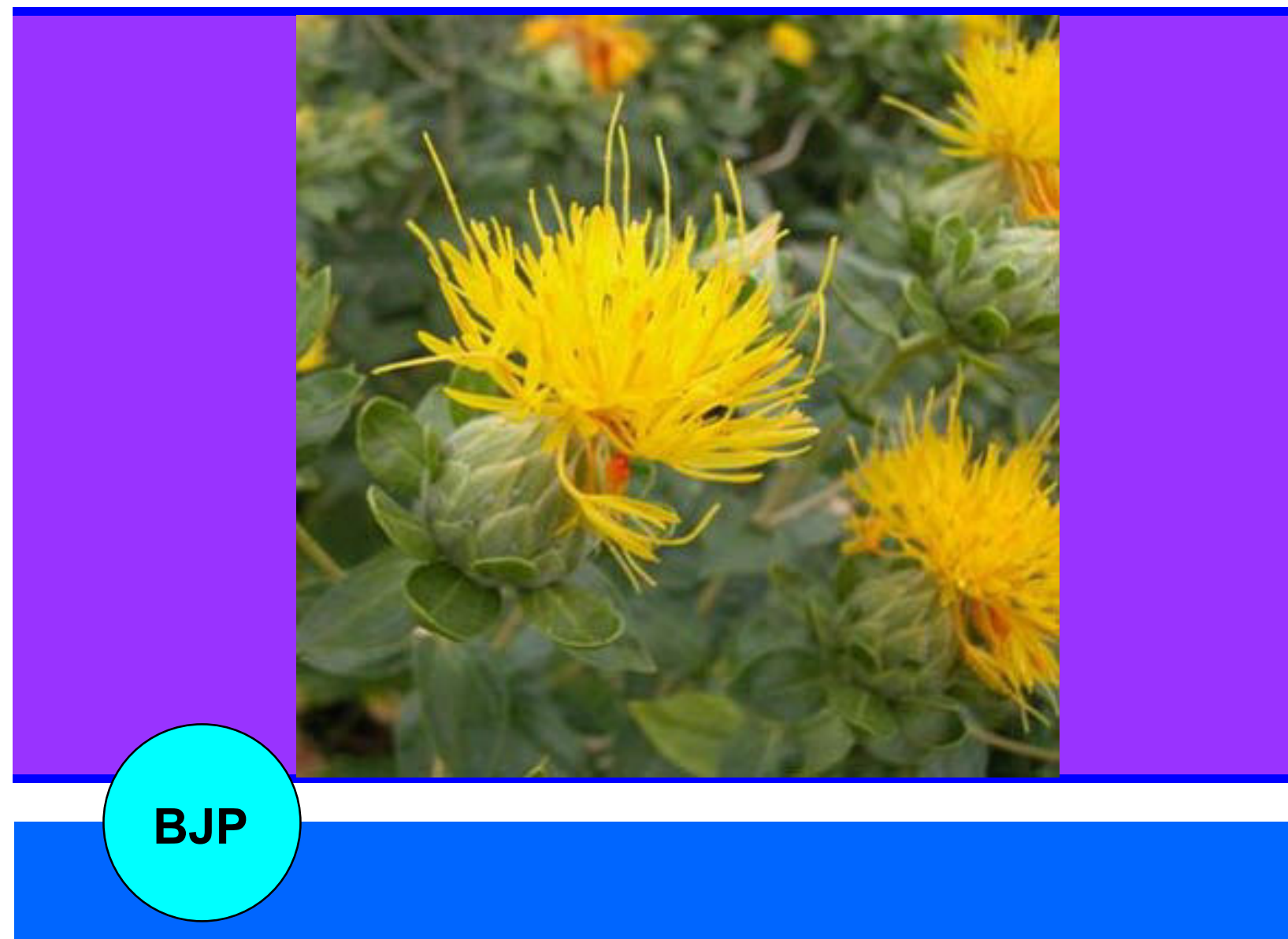

Bangladesh Journal of Pharmacology

Research Article

Effect of safflower yellow on platelet activating factor mediated platelet activation in patients with coronary heart disease 
Abstracted/indexed in Academic Search Complete, Asia Journals Online, Bangladesh Journals Online, Biological Abstracts, BIOSIS Previews, CAB Abstracts, Current Abstracts, Directory of Open Access Journals, EMBASE/Excerpta Medica, Google Scholar, HINARI (WHO), International Pharmaceutical Abstracts, Open J-gate, Science Citation Index Expanded, SCOPUS and Social Sciences Citation Index;

ISSN: $1991-0088$

\title{
Effect of safflower yellow on platelet activating factor mediated platelet activation in patients with coronary heart disease
}

\author{
Damin Huang, Yingmin Lu, Xiaohan Luo, Laixin Shi, Jinchun Zhang, Junxian Shen, \\ Minmin Bao, Lei Song, Caiwen Wei, Hongsong Li and Zhihua Li
}

Department of Cardiology, Xinhua (Chongming) Hospital of Shanghai Jiaotong University, Shanghai 202150, China.

\begin{tabular}{|c|c|}
\hline Article Info & \\
\hline Received: & 2 July 2012 \\
\hline Accepted: & 3 July 2012 \\
\hline Available Online: & 10 July 2012 \\
\hline DOI: $10.3329 /$ bjp.v & 094 \\
\hline Cite this article: & \\
\hline Huang D, Lu Y, Lu & hi L, Zhang J, \\
\hline Shen J, Bao M, Son & ei C, $\mathrm{Li} \mathrm{H}, \mathrm{Li}$ \\
\hline Z. Effect of safflow & low on plate- \\
\hline $\begin{array}{l}\text { let activating facto } \\
\text { activation in patie }\end{array}$ & $\begin{array}{l}\text { iated platelet } \\
\text { ith coronary }\end{array}$ \\
\hline $\begin{array}{l}\text { heart disease. Ban } \\
\text { col. 2012; 7: 140-44. }\end{array}$ & h J Pharma- \\
\hline
\end{tabular}

\begin{abstract}
The platelet aggregation and 5-HT release by washed platelet from coronary heart disease patients following platelet activating factor (PAF) treatment were detected by turbidimetry and O-phthalaldehyde assay. The free calcium concentration in the platelets was measured with the fura-2/AM probe fluorescent technique. Results showed safflower yellow could inhibit the PAF induced washed platelet aggregation and 5-HT release, which were in a safflor-yellow-dose dependent manner. When the PAF was $2.0 \times 10-9 \mathrm{~mol} / \mathrm{L}$, the inhibition rate of platelet aggregation was $26.2,41.3,58.1,81.2 \%$, and the inhibition rate of 5-HT release was 3.7, 11.9, 29.9 and $54.4 \%$ after treatment with safflower yellow at $0.2,0.4,0.8$ and $1.7 \mathrm{~g} / \mathrm{L}$, respectively. The study concludes safflower yellow can inhibit the PAF induced platelet aggregation, 5 -HT release by platelets and elevation of free calcium in platelets.
\end{abstract}

\section{Introduction}

Safflower is the dry flower of Cathamus tinctorius L which has been used in traditional Chinese medicine for promoting blood circulation for removing blood stasis. To date, it has been applied in the treatment of trauma, gynecological diseases, cardiovascular diseases and pulmonary fibrosis (Wang et al., 2011). More than 200 compounds have been isolated from safflower including flavonoids, phenylethanoid glycosides, coumarins, fatty acids, steriods and safflower polysaccharides. Among these compounds, safflower yellow (SY) is the effective part of the aqueous extract of safflower (Fan et al., 2009). Hydroxy safflower yellow A (HSYA) is an important active ingredient of SY and safflower. The SY for injection has been used to treat myocardial ischemia in clinical at $80 \mathrm{mg} / \mathrm{d}$ (Wang et al., 2011).

Safflower yellow is a common traditional Chinese medicine for promoting blood circulation for removing blood stasis. It has been used in the treatment of blood circulation disorders such as coronary heart disease. In these diseases, hypercoagulable state characterized by platelet activation, coagulation and alteration in fibrinolytic activity can be found at any stage, which attribute to the coronary artery spasm, myocardial ischemia and myocardial infarction (Görög et al., 1995). In recent years, studies have confirmed that TAT, GM P -140 and tpA can serve as markers for thrombosis and prothrombotic state and have been used in clinical practice. In the development of these diseases, platelet activation is frequently found accompanied by increase in free calcium in the platelets. Platelet activating factor (PAF) is an inflammatory cytokine synthesized in multiple cells and has been found to be the most potent inducer of platelet aggregation (Penna et al., 2011). Study has confirmed than PAF involves in the occurrence and development of numerous human disease and plays important roles in the pathophysiology of some cardiovascular and cerebrovascular diseases (Shen and Li, 1991). Clinically, aqueous extract of safflower is the main drug used and safflower yellow is the major aqueous-soluble ingredient and belongs to 
chalcone ketone (Wang et al., 2011; Zhou, 1993). In the present study, the effect of safflower yellow on the PAF induced platelet aggregation, 5-HT release by platelets and the free calcium in platelets aiming to explore the mechanism of safflower yellow to promote blood circulation for removing blood stasis.

\section{Materials and Methods}

\section{Drugs and reagents}

Carthamus tinctorius was purchased from the pharmaceutical material and drug corporation of Haidian District, Beijing and identified by Prof. J. S. Li, Department of Chinese Medicine Identification, Beijing University of Traditional Chinese Medicine. Safflower yellow was prepared from aqueous abstract of $C$. tinctorius followed by silicon gel adsorption (Patent number: 98125121. 8) at the Department of Chinese Medicine Identification, Beijing University of Traditional Chinese Medicine. PAF (Sigma), Ginkolide (Total terpene lactone: $81.4 \%$; ginkolide A: $39.6 \%$; ginkolide B: $39.9 \%$; ginkolide C: $2.0 \%$ ), $0.05 \%$ O- phthalaldehyde in $1 \%$ absolute ethanol and $10 \mathrm{~mol} / \mathrm{L} \mathrm{HCl}$ (OPT; Chemical Reagent Company of Beijing; stored in brown bottle at $4^{\circ} \mathrm{C}$ and used within 1 month), $60 \mathrm{mg} / \mathrm{L}$ 5-HT creatinine sulfate (Sigma; $4^{\circ} \mathrm{C}$ ), DMSO and Fura-2/AM (Sigma) were used in the present study. $500 \mu \mathrm{g} / \mathrm{L} 5-\mathrm{HT}$ creatinine sulfate was prepared immediately before experiment. Fura-2/AM was dissolved in DMSO at a final concentration of $1 \mathrm{mmol} / \mathrm{L}$ and stored in dark at $30^{\circ} \mathrm{C}$. The remaining reagents had analytic purity.

\section{General information}

Five male patients with coronary heart disease who were aged $48 \pm 7$ years were recruited. The coronary heart disease was diagnosed according to the criteria developed by World Health Organization. Thrombosis and bleeding disorders were excluded from these patients. The study has been approved by the ethics committee of Xinhua Hospital and written inform consent has been obtained from each patient.

\section{Instruments}

Chrono-log platelet aggregation analyzer and Hitachi $\mathrm{F}$ -2000 Fluorescence spectrophotometer (Japan) were used in the present study.

\section{Preparation of washed platelets}

Blood was collected from the artery and mixed with ACD solution $(85 \mathrm{mmol} / \mathrm{L}$ sodium citrate, $71 \mathrm{mmol} / \mathrm{L}$ citrate acid, $110 \mathrm{mmol} / \mathrm{L}$ glucose) at a ratio of 1:6 (v/v) for anti-coagulation. Centrifugation was done at $30 \times \mathrm{g}$ for $5 \mathrm{~min}$ and supernatant (plasma) rich in platelets was collected followed by centrifugation at $500 \times \mathrm{g}$ for $5 \mathrm{~min}$. The supernatant was removed and the platelets in the sediments were suspended in calcium free Tyrode's solution (pH6.5, $2.62 \mathrm{mmol} / \mathrm{L} \mathrm{KCl}, 1.0 \mathrm{mmol} \cdot \mathrm{L} \mathrm{MgCl}_{2}$, $137 \mathrm{mmol} / \mathrm{L} \mathrm{NaCl}, 12 \mathrm{mmol} / \mathrm{L} \mathrm{NaHCO}_{3}, 5.6 \mathrm{mmol} / \mathrm{L}$ glucose, $0.25 \%$ bovine serum albumin (BSA) which was the washed coronary heart disease platelet. TTBSA was used to adjust the platelet concentration at $2.5 \times 1011 / \mathrm{L}$. (LaLau et al., 1984)

\section{Detection of platelet aggregation}

Three groups were included: Normal saline group, ginkgolide group and Safflower yellow group. In brief, $180 \mu \mathrm{L}$ of washed platelet was mixed with normal saline, ginkgolide or safflower yellow $(10 \mu \mathrm{L}$ in each group) which was then mixed in $1 \times 10-8 \mathrm{~mol} / \mathrm{L}$ PAF$0.25 \%$ BSA calcium free Tyrode's solution $(10 \mu \mathrm{L})$. The curve was delineated at $37^{\circ} \mathrm{C}$. The platelet aggregation rate and inhibition rate (I) of platelet aggregation rate were calculated aiming to observe the suppressive effect of Safflower yellow on the platelet aggregation. I $=\left(100 \%\right.$ - platelet aggregation rate treatment $_{\text {(PNS) }}$ platelet aggregation $_{\text {normal saline) }} \times 100 \%$.

\section{Detection of 5-HT release by platelets}

The platelet aggregation was prepared. The mixture was centrifuged at $500 \times \mathrm{g}$ for $10 \mathrm{~min}$. The supernatant $(0.3 \mathrm{~mL})$ was collected for detection. At the same time, $0.3 \mathrm{~mL}$ of $500 \mu \mathrm{g} / \mathrm{L} 5$-HT standard solution was also used for detection. In each tube, $0.7 \mathrm{~mL}$ of TTBSA and $0.3 \mathrm{~mL}$ of $100 \%$ trichloroacetic acid were added followed by centrifugation at $1500 \times \mathrm{g}$ for $20 \mathrm{~min}$. The supernatant was collected and mixed with OPT HCL followed by being boiled for $10 \mathrm{~min}$ and kept on ice for $10 \mathrm{~min}$. Then, $2 \mathrm{~mL}$ of chloroform was added followed by centrifugation at $1500 \times \mathrm{g}$ for $20 \mathrm{~min}$. The supernatant $(1 \mathrm{~mL})$ was collected and the fluorescence was measured at excitation wavelength of $360 \mathrm{~nm}$ and emission wavelength of $475 \mathrm{~nm}$. The fluorescence value (F) was recorded and F475/360 was calculated. In the safflower yellow group, safflower yellow at each concentration was diluted with TTBSA and $0.3 \mathrm{~mL}$ of this safflower yellow solution was used as a control. The actual F475/360 of safflower yellow was subtracted by that of corresponding control serving as the adjusted F475/360 of safflower yellow (Hou et al., 1984).

\section{Detection of free calcium in platelets}

The platelet sediments were collected and mixed in Hepes buffer ( $\mathrm{pH} 7.4,145 \mathrm{mmol} / \mathrm{L} \mathrm{NaCl}, 5 \mathrm{mmol} / \mathrm{L}$ $\mathrm{KCl}, 1 \mathrm{mmol} / \mathrm{L} \mathrm{MgSO}_{4}, 5 \mathrm{mmol} / \mathrm{L}$ glucose, $10 \mathrm{mmol} / \mathrm{L}$ Hepes, $0.5 \mathrm{mmol} / \mathrm{L} \mathrm{Na}_{2} \mathrm{HPO}_{4}$, and $0.25 \%$ BSA). The platelet concentration was adjusted at 3. $5 \times 1011 / \mathrm{L}$. Then, Fura- 2/AM was added to platelet suspension at a final concentration of $2 \mu \mathrm{mol} / \mathrm{L}$. This mixture was under continuous shaking at $37^{\circ} \mathrm{C}$ for $40 \mathrm{sec}$. The platelets were washed with Hepes buffer twice followed by centrifugation at $500 \times \mathrm{g}$ for $5 \mathrm{~min}$. Then, the platelets were re-suspended in Hepes buffer which was the Fura -2 loading platelet suspension. Three groups were included: PAF group, ginkgolide group and Safflower 
Table I

Effect of safflower yellow on PAF $\left(2.0 \times 10_{-9} \mathrm{~mol} / \mathrm{L}\right)$ induced platelet aggregation

\begin{tabular}{|llll|}
\hline Group & \multicolumn{1}{c|}{ Concentration $(\mathrm{pg} / \mathrm{L})$} & \multicolumn{1}{c|}{ Aggregation rate/P\% } & Inhibition rate/P\% \\
\hline Normal saline & & $85.1 \pm 5.0$ & $59.1^{\mathrm{a}}$ \\
Ginkgolide & $2.1 \times 10-3$ & $31.1 \pm 2.42^{\mathrm{a}}$ & $91.3^{\mathrm{a}}$ \\
Ginkgolide & $2.1 \times 10-2$ & $5.4 \pm 1.71^{\mathrm{a}}$ & $26.2^{\mathrm{a}}$ \\
Safflower yellow & $2.1 \times 10-1$ & $59.1 \pm 5.8^{\mathrm{a}}$ & $41.3^{\mathrm{a}}$ \\
Safflower yellow & $4.1 \times 10-1$ & $60.8 \pm 4.2^{\mathrm{a}}$ & $58.1^{\mathrm{a}}$ \\
Safflower yellow & $7.9 \times 10-1$ & $31.6 \pm 8.1^{\mathrm{a}}$ & $81.2^{\mathrm{a}}$ \\
Safflower yellow & 1.6 & $20.8 \pm 2.1^{\mathrm{a}}$ & \\
\hline
\end{tabular}

$\left(\mathrm{n}=3,{ }^{x} \pm \mathrm{s}\right) ;{ }^{a} \mathrm{p}<0.05$ vs normal saline group

\section{Table II}

Effect of safflower yellow on PAF $(2.0 \times 10-9 \mathrm{~mol} / \mathrm{L})$ induced 5 -HT release by platelets

\begin{tabular}{|llllc|}
\hline Group & Concentration $(\mathrm{pg} / \mathrm{L})$ & Fluorescence value & 5-HT/Pmg L - & 5-HT release inhibition rate/\% \\
\hline Normal saline & & $482 \pm 23.5$ & $2.7 \pm 0.1$ & $77.1^{\mathrm{a}}$ \\
Ginkgolide & $2.0 \times 10-3$ & $121 \pm 9.3^{\mathrm{a}}$ & $0.6 \pm 0.2^{\mathrm{a}}$ & $3.7^{\mathrm{b}}$ \\
Safflower yellow & $2.1 \times 10-1$ & $456 \pm 7.2^{\mathrm{b}}$ & $2.2 \pm 0.1^{\mathrm{b}}$ & $11.9^{\mathrm{b}}$ \\
Safflower yellow & $4.2 \times 10-1$ & $420 \pm 31.3^{\mathrm{b}}$ & $2.2 \pm 0.1^{\mathrm{b}}$ & $29.9^{\mathrm{a}}$ \\
Safflower yellow & $8.5 \times 10-1$ & $312 \pm 46.4^{\mathrm{a}}$ & $1.5 \pm 0.03^{\mathrm{a}}$ & $54.4^{\mathrm{a}}$ \\
Safflower yellow & 1.6 & $200 \pm 3.3^{\mathrm{a}}$ & $1.2 \pm 0.02^{\mathrm{a}}$ & \\
\hline
\end{tabular}

$(\mathrm{n}=3, x \pm \mathrm{s}) ; \mathrm{a} \mathrm{p}<0.01 ; \mathrm{b} \mathrm{p}>0.05$ vs normal saline group

yellow group. In brief, $0.56 \mathrm{~mL}$ of Fura-2 loading platelet suspension was mixed with $6 \mu \mathrm{L}$ of $0.1 \mathrm{~mol} / \mathrm{L}$ $\mathrm{CaCl}_{2}$. Then, normal saline, ginkgolide and safflower yellow at different concentration were added to corresponding tubes $(10 \mu \mathrm{L}$ in each group) followed by incubation at $37^{\circ} \mathrm{C}$ for $3 \mathrm{~min}$. Then, fluorescence was measured at excitation wavelength of 340 or $380 \mathrm{~nm}$ and emission wavelength of $510 \mathrm{~nm}$. The curve was delineated over time and $24 \mu \mathrm{L}$ of $2 \times 10-8 \mathrm{~mol} / \mathrm{L} \mathrm{PAF}$ $20.25 \%$ BSA calcium free Tyrode's solution was added at 20 30 sec after detection. The curve was recorded over time. The F340 and F380 were measured at rest and after PAF treatment. Safflower yellow might interfere with the fluorescence leading to the reduction of fluorescence value and thus the fluorescence value in the safflower yellow group was adjusted as follows. $\Delta F 340=F 340$ normal saline $-F 340$ safflower yellow, $\Delta \mathrm{F} 380=\mathrm{F} 380$ normal saline $-\mathrm{F} 380$ safflower yellow, F340 adjustment $=$ Fp340 safflower yellow $+\Delta$ F340 and F380 adjustment $=\mathrm{Fp} 380_{\text {safflower yellow }}+\Delta \mathrm{F} 380$. Following Fura-2 loading, fluorescence was measured at excitation wavelength of 340 and $380 \mathrm{~nm}$ and emission wavelength of $510 \mathrm{~nm}$, and the F340/380 was parallel to the free calcium in the platelets. In the present study, the F340/380 in the normal saline group served as the free calcium in the platelets at rest. After treatment with
PAF, the Fp340/Fp380 was measured and after treatment with PAF + safflower, the adjusted F340/380 was calculated to reflect the effect of safflower yellow. In the present study, experiment was done twice and means were calculated (Fang et al., 1993).

\section{Results}

When the PAF concentration was $2.0 \times 10-9 \mathrm{~mol} / \mathrm{L}$, the aggregation rate was $85.1 \%$ and safflower yellow could inhibit the PAF induced platelet aggregation in a dose dependent manner. When the ginkgolide concentration was $2.10 \times 10-2 \mathrm{pg} / \mathrm{L}$, the inhibition rate of platelet aggregation was $91.3 \%$ and when the safflower yellow was at $1.6 \mathrm{pg} / \mathrm{L}$, the inhibition rate of platelet aggregation was $81.2 \%$ (Table I).

Following detection of platelet aggregation, the 5-HT content in the supernatant was measured. Results showed safflower yellow could inhibit the PAF induced 5-HT release in a dose dependent manner (Table II).

In the normal saline group, $\mathrm{F}_{\mathrm{r} 340} / \mathrm{F}_{\mathrm{r} 380}$ served as the free calcium content in the platelets at rest. The $\mathrm{F}_{\mathrm{p} 340} / \mathrm{F}_{\mathrm{p} 380}$ was measured as the free calcium content in the platelets after PAF treatment. The adjusted $\mathrm{F}_{\mathrm{p} 340} / \mathrm{F}_{\mathrm{p} 380}$ 
Table III

\begin{tabular}{|c|c|c|}
\hline \multicolumn{3}{|c|}{$\begin{array}{c}\text { Effect of safflower yellow on PAF }\left(8.0 \times 10_{-10} \mathrm{~mol} / \mathrm{L}\right) \\
\text { induced elevation of free calcium in Fura-2 loading } \\
\text { platelets }\end{array}$} \\
\hline Group & $\begin{array}{l}\text { Concentration/ } \\
\times 10-3 \mathrm{~g} \cdot \mathrm{L}-1\end{array}$ & $\begin{array}{c}\text { adjusted } \mathrm{F}_{\mathrm{p} 340} / \\
\mathrm{F}_{\mathrm{p} 380}\end{array}$ \\
\hline Normal saline & & $5.8 \pm 0.02^{a}$ \\
\hline Ginkgolide & 6.7 & $3.0 \pm 0.2^{\mathrm{a}}$ \\
\hline Safflower yellow & 4.3 & $3.6 \pm 0.8^{a}$ \\
\hline Safflower yellow & 6.1 & $3.2 \pm 0.2^{\mathrm{a}}$ \\
\hline Safflower yellow & 10.1 & $3.2 \pm 0.4^{a}$ \\
\hline Safflower yellow & 16.2 & $2.5 \pm 0.6^{\mathrm{a}}$ \\
\hline Safflower yellow & 26.52 & $2.4 \pm 0.6^{a}$ \\
\hline
\end{tabular}

$(\mathrm{n}=3, x \pm \mathrm{s}) ;{ }^{\mathrm{a}} \mathrm{p}<0.01 ; \mathrm{b} \mathrm{p}>0.05 \mathrm{vs}$ normal saline group

served as the free calcium content in the platelets after safflower yellow treatment. Results showed, when the safflower yellow concentration was 4.3, 6.1, 10.1, 16.2 and $26.5 \mathrm{mg} / \mathrm{L}$, the adjusted Fp340/Fp380 was 3.60, $3.21,3.23,2.45$ and 2.43 , respectively. This result revealed safflower yellow could inhibit the PAF induced elevation of free calcium in the platelets (Table III).

\section{Discussion}

In China, numerous studies have conducted to investigate the clinical application and pharmacological effects of safflower yellow. Studies have confirmed that 1) safflower yellow can inhibit thrombosis, dilate blood vessels and combat with platelet aggregation to improve cerebral circulation; 2) safflower yellow can combat with free radicals and oxygenation, elevate the activities of superoxide dismutase and glutathione peroxidase, inhibit the lipid peroxidation and reduce the degradation of phospholipid in the membrane which then protect cells and promote cellular metabolism; 3) safflower yellow can reduce the levels of cholesterol and triglyceride and improve the blood viscosity; 4) safflower yellow can exert neuroprotective effect which prevent ischemia/reperfusion injury, improve neurological function and attenuate brain edema (Jiang, 2011).

Safflower contains safflower yellow and carthamin. Safflower yellow can be typed into É, $\hat{E}$ and $\ddot{E}$ among which safflower yellow of Ë type has the most potent activity and the major ingredient. Safflower yellow of $\ddot{E}$ type can inhibit platelet aggregation, elevate the activity of fibrinolytic protease in rats and confer suppressive effect on thrombosis in vitro. Safflower can improve the hypercoagulable state in different ways: 1) safflower can inhibit the activity of platelets to affect the adherence, aggregation of platelets and release of inflammatory factors by platelets, which inhibit the initial step in the thrombosis; thus safflower yellow can inhibit the formation of arterial thrombus the major ingredient of which is platelet. Study has confirmed that safflower yellow can significantly inhibit ADP induced platelet aggregation; 2) anti-coagulation effect: Thrombin is a key factor in the thrombosis. The reduction of thrombin avoids the thrombosis due to influence of thrombin on platelets and coagulation; reduce the influence of thrombin on endothelial cells and decline the generation and release of plasminogen activator inhibitor (PA I/1) by endothelial cells; 3) protects endothelial cells. The reduction of thrombin and increase in tpA atenuate the damage of thrombin and fibrin to endothelial cells, which is required to be further studied.

In our study, results demonstrated that safflower yellow exerted suppressive effect on PAF induced platelet activation, and removal of safflower yellow could reverse this suppressive effect. These findings suggested that saflower yellow is a major aqueous soluble ingredient exerting anti-PAF effect. PAF is the most potent anti-platelet activation factor so far. PAF can be synthesized by platelets, white blood cells, endothelial cells and other cells and has wide biological activities. There is evidence showing that PAF is an important mediator in numerous diseases and closely related to ischemia-reperfusion injury, coronary heart disease, atherosclerosis, cerebrovascular diseases and other ischemia related cardiovascular and cerebrovascular diseases (Penna et al., 2011). In these diseases, the platelet aggregation is active and some thrombogenic factors including TXA2, $32 \mathrm{TG}, 5-\mathrm{HT}$, etc are released leading to the tendency to thrombosis. PAF can activate phospholipase C (PLC) and catalyze the hydrolysis of phosphatidyl inositol into diacylglycerol (DAG) and inositol triphosphate (IP3). IP3 can promote the release of calcium via the dense pipeline system and facilitate the influx of calcium resulting in increase in intracellular calcium (Logan et al., 2000; Moriguchi et al., 2010). The intracellular calcium involves in the regulation of platelet function. Calcium can act as an initiator to activate the platelet aggregation and release of mediators. The increase in intracellular calcium activates platelets promoting their aggregation and subsequent circulation disorder. Our results demonstrated that safflower yellow could inhibit the PAF induced influx of calcium which was attributed to the suppression of platelet activation and subsequent cardiovascular protection. This is also an important strategy to the improvement of ischemia related cardiovascular and cerebrovascular diseases. Our findings demonstrated that safflower yellow could inhibit PAF induced platelet activation and suppress platelet aggregation, release reaction and increase in intracellular free calcium. Ginkgolide, an inhibitor of PAF receptor, has similar activity to safflower yellow. Thus, we speculated that safflower yellow might act on the membrane of platelet and competitively inhibit the binding bet- 
ween PAF and its receptor which antagonizes the bioactivity of PAF. However, this hypothesis is required to be further confirmed in future studies. Safflower yellow is a common traditional Chinese medicine for promoting blood circulation for removing blood stasis. It has been found to promote menstrualtional flow and remove stasis to ease pain. Clinically, safflower has been used in the treatment of CHD, angina, cerebral thrombosis and vascular diseases. Generally, aqueous extract of safflower is used. Thus, the biological activity of safflower might be related to the ability of safflower yellow to antagonize PAF and improve the platelet function. Safflower has favorable adaptability, is widely distributed and easy to grow. The extraction of safflower yellow is simple. Thus, safflower yellow has promise in clinical application. Some studies have reported the application of safflower yellow in the treatment of ischemia disease. To elucidate the anti-PAF effect of safflower yellow and to develop anti-ischemic drugs have important economic and social benefit.

\section{References}

Fan L, Zhao HY, Xu M, Zhou L, Guo H, Han J, Wang BR, Guo DA Qualitative evaluation and quantitative determination of 10 major active components in Carthamus tinctorius L. by high-performance liquid chromatography coupled with diode array detector. J Chromatogr A. 2009; 1216: 2063-70.

Fang X, Tang XL, Guo ZG. Detection of free calcium in platelets with Fura-2 fluorescence ratio. J Hunan Med Univ. 1993; 18: 209-12.

Görög P, Ridler CD, Rees GM, Kovacs IB. Evidence against hypercoagulability in coronary artery disease. Thromb Res.
1995; 79: 377-85.

Hou YS, Yang SH, Liu ZF. Fluorescence method for the detection of 5-HT in platelets and plasma. J Chin Med Univ. 1984; 13: 44-50.

Jiang H. Pharmacological effects and clinical observation of safflower yellow. J Chin Trad Chin Med Inform. 2011; 3: 205.

LaLau Keraly C, Delautier D, Delebassee D. Inhibition by ticlopidine of PAF acether induced in vitro aggregation of rabbit and human platelets. Thromb Res. 1984; 34: 463-67.

Logan JE, Roudebush WE. Platelet-activating factor increases intracellular calcium levels in preimplantation stage embryos. Early Pregnancy. 2000; 4: 30-38.

Moriguchi S, Shioda N, Yamamoto Y, Fukunaga K. Plateletactivating factor-induced synaptic facilitation is associated with increased calcium/calmodulin-dependent protein kinase II, protein kinase $C$ and extracellular signal-regulated kinase activities in the rat hippocampal CA1 region. Neuroscience 2010; 166: 1158-66.

Penna C, Bassino E, Alloatti G. Platelet activating factor: the good and the bad in the ischemic/reperfused heart. Exp Biol Med (Maywood). 2011; 236: 390-401.

Shen YZ, Li CL. Pathophysiological activity of platelet activation factor. Prog Physiol Sci. 1991; 22: 346-48.

Wang L, Jin M, Zang BX, Wu Y. Inhibitory effect of safflower yellow on pulmonary fibrosis. Biol Pharm Bull. 2011; 34: 51116.

Wang L, Long ZJ, Shi H, Chen M. Effect of Xinkang Injection on PGI_2/TXA_2 and platelet aggregation in a rat myocardial ischemia-reperfusion model. Chin Tradit Patent Med. 2011; 04: 277-79.

Zhou LG. Edible natural pigments and their extraction and application. Jinan, Shandong Science and Technology Press, 1993, pp 158-60. 